\title{
Técnicas de diafanização para estudo da anatomia de dentes humanos
}

\author{
Diaphanization techniques for studying the anatomy of human teeth \\ Técnicas de clarificación para estudiar la anatomía de los dientes humanos
}

Recebido: 13/12/2021 | Revisado: 21/12/2021 | Aceito: 29/12/2021 | Publicado: 06/01/2022

Cleiton Rone dos Santos Lima

ORCID: http://orcid.org/0000-0003-3559-1472

Universidade de Pernambuco, Brasil

E-mail: cleitonrone@live.com

Luiza Vanessa de Lima Silva

ORCID: http://orcid.org/0000-0003-4598-0586

Universidade de Pernambuco, Brasil

E-mail: luizaenfermeira@ hotmail.com

Marcia Jasmini Sidartha da Silva Fernandes

ORCID: http://orcid.org/0000-0001-7594-7424

Universidade de Pernambuco, Brasil

E-mail: fernandes.marcia090@ hotmail.com

Inalda Maria de Oliveira Messias

ORCID: http://orcid.org/0000-0001-8699-3717

Universidade de Pernambuco, Brasil

E-mail: inalda.messias@upe.br

Rosane Jamille de Oliveira Araújo

ORCID: http://orcid.org/0000-0002-6462-2541

Universidade Federal Rural de Pernambuco, Brasil E-mail: jamille.rosane@gmail.com

Mônica Simões Florêncio

ORCID: http://orcid.org/0000-0002-8548-1725 Universidade de Pernambuco, Brasil E-mail: monica.simoes@upe.br

João Ferreira da Silva Filho

ORCID: http://orcid.org/0000-0002-2527-8864 Universidade de Pernambuco, Brasil E-mail: joao.filho@upe.br

Júlio Brando Messias

ORCID: http://orcid.org/0000-0001-6996-974X Universidade de Pernambuco, Brasil E-mail: julio.messias@upe.br

\begin{abstract}
Resumo
A morfologia interna dos canais radiculares difere-se da anatomia externa da raiz dentária, possuindo diversas variações ainda pouco descritas na literatura. A diafanização é um método eficaz, simples e rápido para a análise e preservação de estruturas ou tecidos. Por ser de fácil emprego e baixo custo, constitui-se em um método prático para o estudo da topografia do endodonto. Este estudo constitui-se em uma revisão integrativa da literatura, cujabusca foi realizada nas bases de dados SciELO, MEDLINE e LILACS. Foram incluídos artigos de investigação que respondessemà questão norteadora: "quais os métodos utilizados para diafanizar dentes humanos apresentam resultados satisfatórios nas amostras para análise morfológica?". Foram encontrados 106 resultados dos quais 7 possuíam os critérios e foram incluídos nesta revisão. Durante o processo de diafanização executado pelos autores foram utilizados solventes químicos, sendo eles: xilol, salicilato de metila, EDTA e ácido cítrico. A diafanização destaca-se por proporcionar uma análise tridimensional dos dentes, preservando sua morfologia. Essa análise, quando comparada a outras técnicas, como radiografias, apresentouuma visualização mais detalhada. Ademais, estudos adicionais serão necessários para avaliar se outros solventes, como os orgânicos, que não apresentam toxicidade, são viáveis para o processo de diafanização dos dentes, uma vez devem permanecer armazenados no solvente em que foram diafanizados.
\end{abstract}

Palavras-chave: Odontologia; Endodontia; Técnicas histológicas.

\section{Abstract}

The internal morphology of the root canals differs from the external anatomy of the tooth root, with several variations still little described in the literature. Diaphanization is an effective, simple and fast method for the analysis and preservation of structures or tissues. Because it is easy to use and low cost, it is a practical method for studying the topography of the endodontic. This study is an integrative literature review, whose search was carried out in the SciELO, MEDLINE and LILACS databases. Research articles were included that answered the guiding question: 
"which methods used to clear human teeth show satisfactory results in the samples for morphological analysis?" 106 results were found, of which 6 met the criteria and were included in this review. During the diaphanization process, chemical solvents were used by the authors, namely: xylol, methyl salicylate, EDTA and citric acid. Diaphanization stands out for providing a three-dimensional analysis of the teeth, preserving their morphology. This analysis, when compared to other techniques, such as radiographs, presented a more detailed visualization. Furthermore, additional studies will be needed to assess whether other solvents, such as organic ones, which do not present toxicity, are viable for the process of clearing teeth, since they must remain stored in the solvent in which they were cleared.

Keywords: Dentistry; Endodontics; Histological techniques.

\section{Resumen}

La morfología interna de los conductos radiculares se diferencia de la anatomía externa de la raíz del diente, presentando varias variaciones que todavía están mal descritas en la literatura. El aclarado es un método eficaz, sencillo y rápido para analizar y preservar estructuras o tejidos. Debido a que es fácil de usar y de bajo costo, es un método práctico para estudiar la topografía endodóntica. Este estudio es una revisión integradora de la literatura, cuya búsqueda se realizó en las bases de datos SciELO, MEDLINE y LILACS. Se incluyeron artículos de investigación que respondieron a la pregunta orientadora: "¿qué métodos utilizados para limpiar dientes humanos presentan resultados satisfactorios en muestras para análisis morfológico?". Se encontraron 106 resultados, de los cuales 7 cumplieron con los criterios y fueron incluidos en esta revisión Durante el proceso de aclaramiento realizado por los autores se utilizaron solventes químicos, a saber: xilol, salicilato de metilo, EDTA y ácido cítrico. La diafanización destaca por proporcionar un análisis tridimensional de los dientes, conservando su morfología. Este análisis, en comparación con otras técnicas como las radiografías, presentó una vista más detallada. Además, serán necesarios estudios adicionales para evaluar si otros disolventes, como los orgánicos, que no presentan toxicidad, son viables para el proceso de limpieza de dientes, ya que deben permanecer almacenados en el disolvente en el que fueron aclarados.

Palabras clave: Odontología; Endodoncia; Técnicas histológicas.

\section{Introdução}

A diafanização é uma técnica que compõe o processo de preparos histológicos, sendo também utilizada também para a preservação de amostras biológicas. Trata-se de um método eficaz, simples e rápido para a análise e preservação das estruturas ou tecidos estudados. Por ser de fácil emprego ebaixo custo, torna-seum método prático para o estudo do endodonto. Ao tornar o dente transparente, tem-se uma visãotridimensional da cavidade pulpar ao mesmo tempo em que se mantém a integridade morfológica do dente, possibilitando uma análise entre aanatomia interna e externa do mesmo (Vier et al., 2004).

A morfologia interna dos canais radiculares difere-se da anatomia externa da raiz dentária, possuindo diversas variações ainda pouco descritas na literatura. A origem embriológica das ramificações do canal principal demonstra que pode se dar pela fragmentação da bainha radicular de Hertwig antes da formação dentinária, onde a dentinogênese não ocorre no defeito e forma-se um pequeno canal acessório entre a polpa e o periodonto, ou ainda pelo aprisionamento na bainha radicular em proliferação, de vasos que geralmente estão presentes entre a papila e o folículo dentário. Tais ramificações podem variar quanto ao tamanho, ao número e à localização na raiz dentária (Gutierrez; Aguayo, 1995; Anele et al., 2010; Farias-Junior, 2014).

O conhecimento anatômico radicular interno é um dos fatores primordiaispara eficácia do tratamento endodôntico, pois para procedê-lo o profissional tem limitada visão do local onde atua e, muitas vezes, limita-se apenas aos orifícios dos canais (Pécora et al., 1986). A falta de conhecimento da possibilidade das variações anatômicas internas pode levar a falhas na localização e na instrumentação dos canais, resultando, assim, em insucesso no tratamento endodôntico (Galafassi et al., 2007).

A técnica de diafanização permite analisar detalhadamente a anatomia interna dos dentes, uma vez que revela tridimensionalmente os aspectos do sistema de canais, suas curvaturas e interligações, como um processo relativamente simples e rápido, de baixo custo, utilizando substâncias de baixa toxicidade e que não necessita de complexas aparelhagens (Robertson et al., 1980; Fachini; Rossi-Junior; Duarte, 1998; Farias-Junior, 2014).

Nesse contexto, estudos mostraram que a técnica de diafanização permite obter dentes transparentes, revelando uma imagem segura e complexa de um canal, sob o ponto de vista anatômico, para as aplicações clínicas, uma vez que a morfologia 
dos canais radiculares é altamente complexa e variável (Cunha, 1948; Farias-Junior, 2014). Diante do exposto, o presente estudo objetivou identificar, na literatura cientifica disponível, técnicas utilizadas para a diafanização de dentes humanos.

\section{Metodologia}

Este estudo constitui-se em uma revisão integrativa, método que possibilita a união e análise das experiências publicadas sobre a temática, explorando diversas ideias que possam facilitar o diálogo sobre o tema (Souza; Silva; Carvalho, 2010). A questão norteadora para o desenvolvimento da revisão foi: "quais os métodos utilizados para diafanizar dentes humanos apresentam resultados satisfatórios nas amostras para análise morfológica?"

O levantamento dos artigos científicos foi realizado em setembro de 2019 e atualizado em outubro de 2021, por meio da Biblioteca Virtual em Saúde (BVS), nas bases de dados Scientific Electronic Library Online (SciELO), Medical Literature Analysis and Retrieval System Online (MEDLINE) e Literatura Latino-Americana e do Caribe em Ciências da Saúde (LILACS), fazendo uso da combinação das palavras-chave "diafanização" e "dente". Após realizar a busca nas bases, os resultados foram comparados para excluir duplicatas através do uso do software Mendeley.

Foram incluídos estudos cujas metodologias apresentavam a diafanização de dentes, publicados entre janeiro de 2009 e setembro de 2021, disponíveis na íntegra em português, inglês ou espanhol. Cartas, editoriais, comunicações, revisões e entrevistas foram excluídas.

Estudos selecionados e incluídos foram classificados conforme a metodologia de Souza et al. (2010):

Nível I: evidências resultantes da meta-análise de múltiplos estudos clínicos controlados e randomizados;

Nível II: evidências obtidas em estudos individuais com delineamento experimental;

Nível III: evidências de estudos quase-experimentais;

Nível IV: evidências de estudos descritivos (não-experimentais) ou com abordagem qualitativa;

Nível V: evidências provenientes de relatos de caso ou de experiência;

Nível VI: evidências baseadas em opiniões de especialistas.

A combinação geral de dados dos estudos incluídos se realizou através de síntese descritiva.

\section{Resultados e Discussão}

Na primeira fase da seleção dos estudos foram identificadas 106 citações nas bases de dados eletrônicas. Depois de serem removidos os artigos duplicados permaneceram 50 citações. Após uma revisão exaustiva dos títulos e resumos foram excluídas 30 referências por não se enquadrarem nos objetivos desta revisão. A busca manual a partir das listas de referência dos estudos identificados acrescentou dois estudos adicionais. Ao final da filtragem mantiveram-se 22 artigos para a seleção de texto completo (segunda fase). Esse processo levou à exclusão de 15 estudos (Figura 1). No total, foram selecionados sete artigos para a extração de dados e síntese descritiva (Figura 2). A Figura 1 (fluxograma) detalha o processo de identificação, inclusão e exclusão de estudos. 
Figura 1. Fluxograma da busca na literatura e processo de seleção.

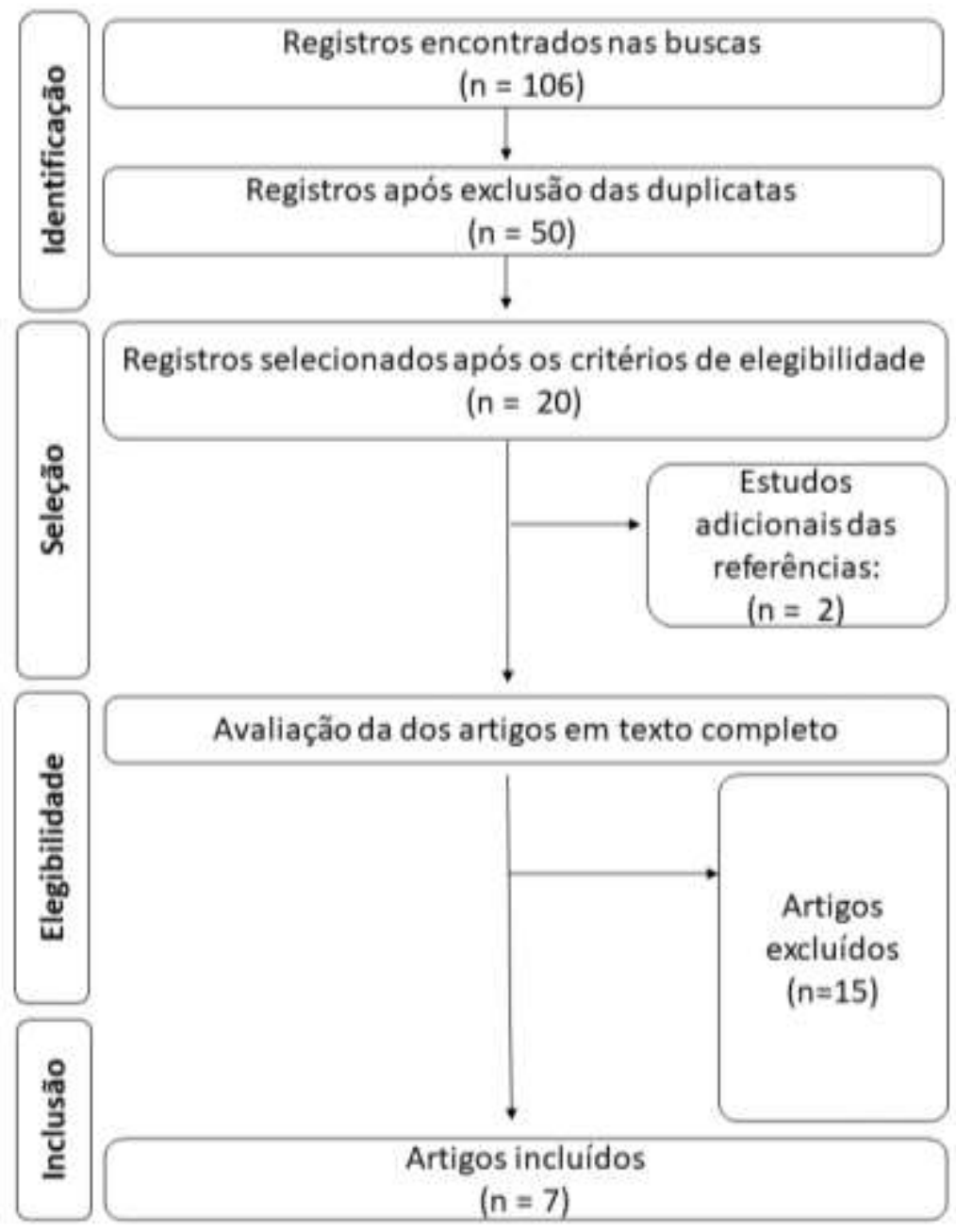

Fonte: Autores

Os estudos incluídos foram publicados em português (Camões et al., 2011; Martin; Azeredo, 2014; Roldi et al., 2016), inglês (Barros et al., 2013; Souza et al., 2015) e espanhol (Fernández; Chacha, 2016; Torres-Obando; Hidalgo, 2018) de 2009 a 2018 e usaram solventes químicos no processo de diafanização. Três estudos (Souza et al., 2015; Roldi et al., 2016; TorresObando; Hidalgo, 2018) apresentaram nível II de evidência e quatro estudos (Camões et al., 2011; Barros et al., 2013; Martin; Azeredo, 2014; Fernández; Chacha, 2016) apresentaram nível III. Para a realização dos experimentos, três estudos (Roldi et al., 2009; Fernández; Chacha, 2016; Torres-Obando; Hidalgo, 2018) utilizaram dentes pré-molares, um estudo utilizou caninos (Martin; Azeredo, 2014), um estudo utilizou primeiros molares (Camões et al., 2011) e um estudo utilizou incisivos inferiores (Souza et al., 2015). Barros et al. (2013) utilizaram diferentes dentes agrupados em molares superiores, molares inferiores, prémolares superiores, pré-molares inferiores, anteriores superiores e anteriores inferiores, permitindo uma maior análise e percepção entre as diferenças anatômicas dos canais.

Quanto aos solventes, dois estudos utilizaram o xilol (Barros et al., 2013; Martin; Azeredo, 2014), quatro estudos utilizaram salicilato de metila (Camões et al., 2011; Souza et al., 2015; Roldi et al., 2016; Fernández; Chacha, 2016) e um dos estudos utilizou EDTA e ácido cítrico (Torres-Obando; Hidalgo, 2018). No Quadro 1 estão resumidas as características descritivas dos estudos. 
Quadro 1. Artigos selecionados e incluídos na revisão.

\begin{tabular}{|c|c|c|c|c|c|}
\hline $\begin{array}{c}\text { Autor (es), } \\
\text { ano }\end{array}$ & $\begin{array}{l}\text { Nível de } \\
\text { evidência }\end{array}$ & Objetivo geral & Amostra (n) & $\begin{array}{c}\text { Solvente } \\
\text { diafanizador }\end{array}$ & Conclusões \\
\hline $\begin{array}{l}\text { Roldi et al., } \\
\qquad 2009\end{array}$ & II & $\begin{array}{c}\text { Comparar o efeito do } \\
\text { hipoclorito de sódio em } \\
\text { diferentes concentrações } \\
\text { associado ou não ao uso do } \\
\text { ultrassom sobre a } \\
\text { permeabilidade da dentina } \\
\text { radicular. }\end{array}$ & $\begin{array}{c}\text { Pré-molares } \\
\text { superiores (30) }\end{array}$ & $\begin{array}{l}\text { Salicilato } \\
\text { de metila }\end{array}$ & $\begin{array}{l}\text { A concentração do hipoclorito de sódio } \\
\text { influenciou na permeabilidade } \\
\text { dentinária, e o hipoclorito a } 5,25 \% \\
\text { apresentou maior capacidade de } \\
\text { aumentar a permeabilidade; o uso do } \\
\text { ultrassom favoreceu o aumento da } \\
\text { permeabilidade, quando se utilizou o } \\
\text { hipoclorito a } 2,5 \% \text {. }\end{array}$ \\
\hline $\begin{array}{l}\text { Camões et al., } \\
2011\end{array}$ & III & $\begin{array}{c}\text { Estudar a frequência do canal } \\
\text { cavo inter-radicularna região } \\
\text { de assoalho pulpar e do } \\
\text { terceiro canal na raiz mesial de } \\
\text { primeiros molares inferiores e } \\
\text { determinar suas morfologias, } \\
\text { através da técnica da } \\
\text { diafanização. }\end{array}$ & $\begin{array}{c}\text { Primeiros molares } \\
\text { inferiores (100) }\end{array}$ & Salicilato de metila & $\begin{array}{l}\text { Conclui-se que o canal cavo } \\
\text { interradicular estava ausente na maioria } \\
\text { das amostras. }\end{array}$ \\
\hline $\begin{array}{l}\text { Barros et } \\
\text { al.,2013 }\end{array}$ & III & $\begin{array}{c}\text { Estudar a influência } \\
\text { da hipercementose na } \\
\text { morfologia radicular e dos } \\
\text { canais radiculares, utilizando } \\
\text { como métodos de observação: } \\
\text { diafanização, radiográfico, } \\
\text { estereomicroscopia e } \\
\text { microscopia óptica. }\end{array}$ & $\begin{array}{c}\text { Molares superiores } \\
\text { (25); molares } \\
\text { inferiores (25); } \\
\text { pré-molares } \\
\text { superiores (25); } \\
\text { pré-molares } \\
\text { inferiores (25); } \\
\text { dentes anteriores } \\
\text { superiores (15); } \\
\text { dentes anteriores } \\
\text { inferiores (15) }\end{array}$ & Xilol & $\begin{array}{c}\text { Os achados apontam para a } \\
\text { possibilidade da existência de uma } \\
\text { anatomia dos canais radicares complexa } \\
\text { no terço apical de dentes com } \\
\text { hipercementose, que, às vezes é } \\
\text { desapercebida pelo exame radiográfico } \\
\text { convencional. }\end{array}$ \\
\hline $\begin{array}{c}\text { Martin e } \\
\text { Azeredo, } 2014\end{array}$ & III & $\begin{array}{l}\text { Analisar o preparo químico- } \\
\text { mecânico dos canais } \\
\text { radiculares realizados por } \\
\text { alunos de graduação em } \\
\text { Odontologia. }\end{array}$ & $\begin{array}{c}\text { Caninos inferiores } \\
(88)\end{array}$ & Xilol & $\begin{array}{l}\text { A diafanização é uma técnica de } \\
\text { visualização acessível e imprescindível } \\
\text { para a evolução do ensino em } \\
\text { Endodontia. }\end{array}$ \\
\hline $\begin{array}{c}\text { Souza et al., } \\
2015\end{array}$ & II & $\begin{array}{c}\text { Avaliar por diafanização a } \\
\text { capacidade de limpeza de } \\
\text { paredes de canais radiculares } \\
\text { achatados preparados com três } \\
\text { instrumentos diferentes. }\end{array}$ & $\begin{array}{l}\text { Incisivos inferiores } \\
\text { (30) }\end{array}$ & Salicilato de metila & $\begin{array}{l}\text { Não houve diferença estatística entre as } \\
\text { técnicas utilizadas quanto à capacidade } \\
\text { de remoção da limpeza das paredes. }\end{array}$ \\
\hline Fernández e & III & Analisar o número de dutos e a & Primeiros & Salicilato de & Os primeiros pré-molares superiores \\
\hline
\end{tabular}




\begin{tabular}{|c|c|c|c|c|c|}
\hline Chacha, 2016 & $\begin{array}{c}\text { presença de um terceiro duto } \\
\text { nos primeiros pré-molares } \\
\text { superiores, utilizando a técnica } \\
\text { de diafanização. }\end{array}$ & $\begin{array}{c}\text { pré-molares } \\
\text { superiores (100) }\end{array}$ & Metila & $\begin{array}{c}\text { apresentaram um, dois e três dutos. A } \\
\text { presença de terceiros dutos teve baixa } \\
\text { prevalência. }\end{array}$ \\
\hline $\begin{array}{c}\text { Torres-Obando e } \\
\text { Hidalgo, 2018 }\end{array}$ & II & $\begin{array}{c}\text { Determinar a microfiltração } \\
\text { apical em ductos selados sem em e } \\
\text { com pré-tratamento de dentina } \\
\text { de EDTA e ácido cítrico }\end{array}$ & Pré-molares (30). & EDTA e Ácido \\
Cítrico & $\begin{array}{c}\text { Os dois tipos de pré-tratamento com } \\
\text { dentina diminuíram eficientemente a } \\
\text { microfiltração apical, sem diferença } \\
\text { estatisticamente significativa. }\end{array}$ \\
\hline
\end{tabular}

Fonte: Autores.

Esta revisão incluiu sete estudos que utilizaram reagentes químicos no processo de diafanização de dentes humanos para estudo da morfologia do sistema de canais radiculares (Roldi et al., 2009; Camões et al., 2011; Barros et al., 2013; Martin; Azeredo, 2014; Fernández; Chacha, 2016; Torres-Obando; Hidalgo, 2018). A literatura aborda que diversas técnicas têm sido aplicadas para o estudo da anatomia dental, principalmente da morfologia interna (Neelakantan et al., 2017). Dentre eles, a técnica de diafanização permitiu classificar frequências de variações anatômicas nos grupos dentários analisados.

De acordo com Pécora (1986) e Silveira (2005), a técnica de diafanização permitiu, pela primeira vez, classificar a anatomia dos canais radiculares. Essa técnica proporciona uma visão tridimensional dos dentes, na qual o espécime fica transparente sem ocorrer, no entanto, quaisquer modificações em sua anatomia. Esse processo é considerado simples e rápido, sendo realizado com substância de baixa toxicidade e sem necessidade de aparelhagens complexas, como investigado por Galafassi et al. (2007).

A anatomia dos canais radiculares é complexa, geralmente composta de ramificações apicais ou coronárias, colaterais, acessórios e comunicações entre canais (Vier et al., 2004).Martin e Azeredo (2014) utilizaram xilol no processo de diafanização e demonstraram que os achados no exame radiográfico apresentaram limitações na visualização de algumas características anatômicas, como, por exemplo, a permeabilidade dentária, que não pôde ser observada radiograficamente, apesar de esta característica ter sido observada na técnica da diafanização em 95,4\% dos canais radiculares (Martin; Azeredo, 2014).

Roldi et al. (2009) elegeram como método para seu estudo a associação do ultrassom e concentrações de hipoclorito de sódio previamente ao processo de diafanização com salicilato de metila. A concentração do hipoclorito de sódio influenciou na permeabilidade dentinária e o hipoclorito a 5,25\% apresentou maior capacidade de aumentar a permeabilidade (Roldi et al., 2009).

Camões et al. (2011) utilizaram a proposta apresentada pelo protocolo de Pécora et al. (1986) para diafanizar o material de seu estudo e elegeram o salicilato de metila como solvente diafanizador. O processo foi realizado imergindo e mantendo os dentes em recipientes individualizados de vidro, contendo $10 \mathrm{ml}$ de salicilato demetila por duas horas. Os resultados mostraram que o canal cavo interradicular estava ausente na maioria das amostras, estando presente em apenas $3 \%$ das amostras, enquanto o terceiro canal na raiz mesial estava presente em $15 \%$ das amostras.

Fernández e Chacha (2016) usaram a técnica de diafanização para investigar os dutos e a existência de um terceiro duto em primeiros pré-molares superiores. Os autores utilizaram como agente diafanizador o salicilato de metila e observaram que, apesar de uma baixa prevalência em sua amostra, existe a presença de um terceiro duto. Já Souza et al. (2015) realizaram a diafanização com salicilato de metila para analisar a eficiência de diferentes instrumentos endodônticos na remoção de tinta impregnada nas paredes dentinárias de incisivos.

Barros et al. (2013) investigaram a influência da hipercementose na morfologia radicular e do canal radicular, 
utilizando como métodos de observação a radiografia, a estereomicroscopia e a microscopia óptica. Para isso usaram como agente clarificante o xilol e, após análise, a alteração mais distinguível durante a comparação entre dentes com e sem hipercementose foi a alta frequência de deltas apicais naqueles com hipercementose.

Torres-Obando e Araújo (2018) investigaram a microfiltração apical em ductos selados sem e com pré-tratamento de dentina de EDTA e ácido cítrico, que serviram como agentes diafanizadores. A observação foi feita em estereomicroscópio óptico e a microfiltração linear foi medida com um calibrador digital. Os dois tipos de pré-tratamento na dentina diminuíram eficientemente a microfiltração apical.

A diafanização permite, ainda, a observação da forma e topografia dos condutos sem perder a relação com a estrutura dental. Com esse método, obtêm-se dentes translúcidos e perante os dentes diafanizados passa a existir uma imagem segura e complexa de um canal sob o ponto de vista anatômico, adquirindo-se informações essenciais para as aplicações clínicas (Cunha, 1948). De acordo com Aprile (1947), o processo da diafanização não oferece dificuldades. O contraste das cavidades após a coloração e a nitidez de seus contornos facilitam o estudo de sua topografia.

Os principais benefícios do método de diafanização referem-se à agilidade com que todo o processo pode ser concretizado, além de ser econômico. A técnica permite maior valorização da anatomia do canal radicular e é um método bastante eficiente para ser usado em aulas e na análise clínica dos dentes com tratamentos fracassados (Robertson et al., 1980).

\section{Conclusão}

Diversas técnicas têm sido aplicadas para o estudo da anatomia dentária, principalmente da morfologia interna. Dentre essas técnicas, a diafanização se destaca por proporcionar uma análise tridimensional dos dentes preservando sua morfologia. A literatura apresenta que nesse processo são utilizados solventes químicos que possuem toxicidade. Faz-se necessário, ainda, que novas pesquisas sejam realizadas para avaliar se outros solventes, como os orgânicos, que não apresentam toxicidade, são viáveis para este processamento, uma vez que os dentes devem permanecer armazenados no solvente em que foram diafanizados.

\section{Referências}

Anele, J. A.; Silva, B. M.; Baratto-Filho, F.; Haragushiku, G.\& Leonardi, D. P. (2010). Prevalência de foraminas e canais acessórios em região de furca e assoalho pulpar e sua influência na etiologia da lesão endoperiodontal. Odonto, 18(35):106-16.

Aprile, E. C. \&Aprile, H. (1947). Contribuição ao estudo da topografia dos canais radiculares. Rev Assoe Paul Cir Dent, 1:13-6.

Barros, L. A. P.; Pinheiro, B. C.; Azeredo, R. A.; Consolaro, A.\& Pinheiro, T. N. (2013) Root and canal morphology of the apical third of teeth with hypercementosis. Dental Press Endod, 3(3): 49-57.

Camões, I. C. G.; Freitas, L. F.; Santiago, C. N.; Gomes, C. C.; Sambati, G.\&Sambati, S. (2011). Estudo in vitro da frequência do canal cavo inter radicular e do terceiro canal na raiz mesial de molares inferiores. RevOdontolUniv, 23(2): 124-33.

Cunha, E. S. (1948). Diafanização de dentes pelo processo Okumura - Aprile. RevAssoc Paul Cir Dent, 1(6):11-5.

Fachini, E. V. F.; Rossi-Júnior, A. \& Duarte, T. S. (1998). Contribuição ao estudo da técnica da diafanização. RevFacOdontol, 39(1):3-8.

Farias-Junior, J. F. Estudo do sistema de canais radiculares em dentes com hipercementose (Dissertação). Vitória (ES): Universidade Federal do Espírito Santo, Centro de Ciências da Saúde; 2014

Fernández, K. S. \& Chacha, A. M. (2016). Prevalencia de un tercer conducto en primeros premolares superiores mediante diafanización. Odontología, 8(1): 26-32.

Galafassi, D.; Lazaaretti, D. N.; Spazzin, A. O.; Vanni, J. R.\& Silva, S. O. (2007) Estudo da anatomia interna do canal radicular em incisivos inferiores pela técnica da diafanização. RSBO, 1(4):7-11.

Gutierrez, J.H\&; Aguayo, P. (1995). Apical foraminal openings in humans teeth. Number e location. Oral Surg, 79(6):769-77.

Martin, G. \& Azeredo, R.A. (2014). Análise do preparo de canais radiculares utilizando-se a diafanização. Rev. Odontol. UNESP, 43(2):111-8.

Neelakantan, P.; Subbarao, C.; Ahuja, R.\&Subbarao, C.V. (2011). Root and canal morphology of Indian maxillary premolars by a modifed root canal staining technique. Odontology, 99(1):18-21. 
Research, Society and Development, v. 11, n. 1, e27911124695, 2022

(CC BY 4.0) | ISSN 2525-3409 | DOI: http://dx.doi.org/10.33448/rsd-v11i1.24695

Pécora, J. D.;Savioli, R. N.;Vansan, L. P.; Silva, R. G.\& Costa, W. F. (1986).Novo método de diafanizar dentes. RevFac Odontol. Ribeirão Preto, $23(1): 1-5$.

Robertson, D.; Leeb, J.;Mckee, M.\& Brewer, E.(1980). A clearing technique for the study of root canal systems. J Endod, 6(1):421-4.

Roldi, A.; Gagno, A. S.; Barroso, J.; Intra, J. B. G; Intra, T. J. A.; Souza, R. P.\& Ribeiro, F. C. (2009). Avaliação do hipoclorito de sódio associado ou não ao uso do ultrassom sobre a permeabilidade dentinária. RevBrasPesq Saúde, 11(1):11-5

Silveira, L. F. M.; Danesib, V.C.\&Baischc, G.S. (2005). Estudo das relações anatômicas entre os canais mesiais de molares inferiores. Revista de Endodontia Pesquisa e Ensino OnLine, 1(2):16-21.

Souza, M. T.; Silva,M.D.\&Carvalho, R. (2010). Revisão integrativa: o que é e como fazer. São Paulo: Einstein.

Souza, W. A. S. B.; Gonçalves, P. S.; Rasquin, L. C.\& Carvalho, F. B. (2015). Analysis of cleaning capacity of three instrumentation techniques in flattened root canals: diaphanization study. Journal of Dentistry \& Public Health, 6(1).

Torres-Obando, S. A. \& Hidalgo, A. P. D. (2018). Microfltración apical en conductos obturados con y sin pretratamiento dentinario: Estudio in vitro. Odontología, 20(1):50-60.

Vier, F. V.; Reis, M. V.; Mattuella, L. G.; Oliveira, F.; Bozza, K.\&Oliveira, E. P. (2004). Correlação entre o exame radiológico e a diafanização na determinação do número de canais em primeiros pré-molares inferiores com e sem sulco longitudinal radicular. Odont Clin Cient, 3(1):39-48. 